\title{
Dynamic expression of SrGAP2 in cell nuclei and cytoplasm during the differentiation of rat neural stem cells in vitro
}

\author{
QIAN JIAO $^{1}$, LI WANG ${ }^{1}$, ZHICHAO ZHANG $^{1}$, YUANYUAN WANG ${ }^{1}$, \\ HANQI YAN ${ }^{1}$, WEN MA ${ }^{1,2}$, WEILIN JIN ${ }^{3}$, HAIXIA LU ${ }^{1 *}$ and YONG LIU ${ }^{1 *}$ \\ ${ }^{1}$ Institute of Neurobiology, Xi'an Jiaotong University Health Science Centre, Xi'an, Shaanxi 710061; \\ ${ }^{2}$ Department of Genetics and Cell Biology, Ningxia Medical School, Yinchuan, Ningxia 750004; \\ ${ }^{3}$ School of Life Science and Biotechnology, Shanghai Jiao Tong University, Shanghai 200240, P.R. China
}

Received October 10, 2015; Accepted November 25, 2016

DOI: $10.3892 / \mathrm{mmr} .2016 .5795$

\begin{abstract}
Different SLIT-ROBO Rho GTPase-activating proteins (srGAPs) have different levels of expression and diverse functions during neural development. Although srGAP2 is expressed in developmental brain tissue, little is known about its influence on cellular development of the nervous system. In the current study, dynamic expression of endogenous srGAP2 during neural stem cell/progenitor cell (NSC/NPC) differentiation in vitro was investigated in order to elucidate the association between the dynamic expression of srGAP2 and neural development. srGAP2 was expressed in undifferentiated NSCs/NPCs, and differentiated neurons and astrocytes with distinct expression patterns. In conjunction with the differentiation of NSCs/NPCs in vitro, the number of srGAP2 ${ }^{+}$cells markedly reduced. The percentage of srGAP2 ${ }^{+}$ cells in the population of nestin ${ }^{+}$and $\beta$-tubulin $\mathrm{III}^{+}$cells was significantly downregulated while in the population of glial fibrillary acidic protein-positive cells, almost all cells were srGAP2 ${ }^{+}$. srGAP2 was predominantly expressed in the cell nucleus in all cell types. srGAP2 was also weakly expressed in the cytoplasm of nestin ${ }^{+}$and $\beta$-tubulin $\mathrm{III}^{+}$cells at 3 and 7 days in vitro. However levels were gradually downregulated during the process of differentiation and almost disappeared in $\beta$-tubulin $\mathrm{III}^{+}$cells at 14 days. The results from the present study suggest that srGAP2 is involved in regulating NSC/NPC differentiation during neural development. The translocation of srGAP2 in the cytoplasm and cell nucleus in different cell
\end{abstract}

Correspondence to: Professor Haixia Lu or Professor Yong Liu, Institute of Neurobiology, Xi'an Jiaotong University Health Science Centre, 76 Yanta Road, Xi'an, Shaanxi 710061, P.R. China

E-mail: hx101@mail.xjtu.edu.cn

E-mail: liuy5599@mail.xjtu.edu.cn

${ }^{*}$ Contributed equally

Key words: srGAP2, neural stem cells, differentiation, dynamic expression types may function as a director in decisions regarding cell fate.

\section{Introduction}

In mammals, the SLIT-ROBO-GTPase activating protein (srGAP) family consists of four members, srGAP1, 2, 3 and 4 . These proteins were identified as the downstream regulators of the Slit and Robo receptor system and are important in numerous developmental processes in diverse cell types (1-3). During neural development, srGAP1, 2 and 3 are widely expressed in the nervous system and function as multifunctional adaptor proteins involved in neuronal migration, neuronal morphogenesis, neurite outgrowth and synaptic plasticity (1,3-8).

srGAP1 binds to the CC3 conserved cytoplasmic domain of Robol and mediates the blocking effect of Slit on neural progenitor migration via inactivated Ras homologue gene family, member A and cell division control protein 42 homolog (Cdc42), but not Ras-related C3 botulinum toxin substrate 1 (Rac1) (3). srGAP3 regulates Rac1 and Cdc42 and is involved in neuronal morphogenesis and neurite outgrowth $(3,9)$. By contrast, srGAP2 negatively regulates cortical neuronal migration via its IF-BAR domain and promotes neurite outgrowth and branching (7). In addition, the srGAP2 gene has recently been implicated in a severe neurodevelopmental syndrome resulting in infantile epileptic encephalopathy, and srGAP2 knockout mice are prone to epileptic seizures. Similarly, the srGAP3 gene has been associated with mental retardation, and the knockout mice develop lethal hydrocephalus or 'schizophrenia-associated' behaviors $(6,10-13)$. The underlying mechanisms that are involved in the differences between the various srGAPs remain to be elucidated.

NSCs/NPCs are a group of undifferentiated mulitpotent cells which give rise to three predominant types of cells in the nervous system (14-15). They act as valuable tools for the study of neural development. During development, NSCs/ NPCs develop different cellular morphologies whilst retaining their basic properties. Our previous findings have demonstrated that the knockdown of srGAP3 attenuated NSC/NPC survival, proliferation and differentiation and arrested their 

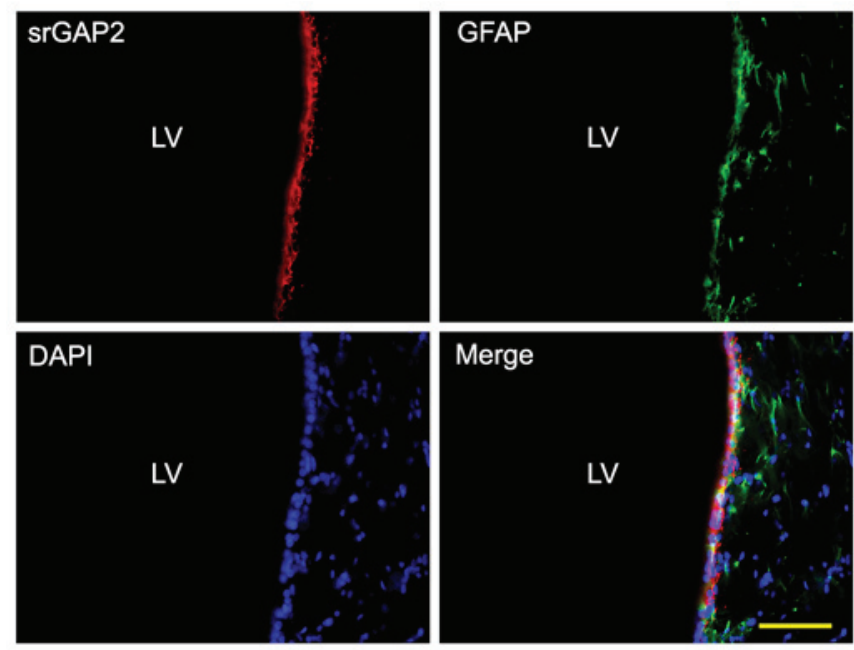

Figure 1. Expression of srGAP2 in SVZ. Cells in the SVZ were double stained by GFAP and srGAP2. Scale bar=100 $\mu \mathrm{m}$. LV, lateral ventricle; srGAP2, SLIT-ROBO Rho GTPase-activity protein 2; GFAP, glial fibrillary acidic protein; SVZ, subventricular zone.

morphological alteration in vitro (16). The influence of srGAP2 on NSCs/NPCs development requires further elucidation.

As srGAP2 was expressed in the developmental rat brain until the 14th postnatal day (17), the present study hypothesizes that, similarly to srGAP3, srGAP2 may exert its effect on neural development via alteration of NSC/NPC proliferation and differentiation. No direct association has yet been indicated between the expression of srGAP2 and the differentiation or morphological maturation of NSCs/NPCs. In addition, the expression pattern of srGAP 2 during postnatal brain development changed dynamically (17). The altered expression in the cytoplasm and nuclei may be associated with its particular function over time. In the present study, the expression of endogenous srGAP2 in NSCs/NPCs during differentiation in vitro was detected, and the proportion of srGAP2 positive cells within the differentiated cell population was analyzed, to elucidate the possible association between the dynamic expression of srGAP2 and the differentiation of NSCs/NPCs.

\section{Materials and methods}

Brian tissue preparation. Six male Sprague-Dawley rats (weight, 250 $\pm 15 \mathrm{~g}$ ) were purchased from the Experimental Animal Center, Xi'an Jiaotong University College of Medicine (Xi'an, China). The environment was controlled with a 12:12-h light/dark cycle, $45-65 \%$ humidity, and room temperature of $20 \pm 2^{\circ} \mathrm{C}$, and the rats had access to food and water ad libitum. All procedures involving animals conformed to the ethical guidelines of the National Institutes of Health (NIH) Guide for the Care and Use of Laboratory Animals (NIH publication no. 85-23, revised 1996), and those set out by the Xi'an Jiaotong University. Rats were fixed by trans-cardiac perfusion with $4 \%$ paraformaldehyde (PFA) in $0.1 \mathrm{M}$ phosphate-buffered saline (PBS) under anesthesia by intraperitoneal injection of $10 \%$ (w/v) chloral hydrate solution (300 mg/kg). Brains were dissected from the skull and then post-fixed with 4\% PFA at $4^{\circ} \mathrm{C}$ overnight. Following gradient elution by sucrose solutions, brain tissue was cut in $15 \mu \mathrm{m}$ sections for immunohistochemical staining.
Culture of rat embryonic NSCs/NPCs. NSCs were isolated from the cerebral cortex of rat embryos on embryonic day 14 and cultured in serum-free growth medium following the protocol of Gage et al (18) and optimized in our laboratory (19). NSC/NPC growth medium contained Dulbecco's modified Eagle's medium/nutrient mixture F-12 (DMEM/F12), 10 ng/ml basic fibroblast grow th factor, $20 \mathrm{ng} / \mathrm{ml}$ epidermal growth factor, $100 \mathrm{U} / \mathrm{ml}$ penicillin, $100 \mu \mathrm{g} / \mathrm{ml}$ streptomycin, $1 \% \mathrm{~N}-2$, and 2\% B-27 supplement (all from Invitrogen; Thermo Fisher Scientific, Inc., Waltham, MA, USA) and $0.4 \mathrm{IU} / \mathrm{ml}$ heparin (Sigma-Aldrich; Merck Millipore, Darmstadt, Germany). Cells were sub-cultured at 5-7 days in vitro (DIV). Upon passage, spheres were trypsinized and mechanically triturated into single cells and replaced at $1 \times 10^{5}$ cells $/ \mathrm{ml}$ and cultured in growth medium as mentioned above.

Induced differentiation of NSCS/NPCs in vitro. Serum was used to induce the spontaneous differentiation of NSCs/ NPCs in vitro. Following passage, a sample of 5,000 NSCs/ NPCs was suspended in $500 \mu \mathrm{l}$ differentiation medium that contained DMEM/F12 with $1 \%$ fetal bovine serum (Invitrogen; ThermoFisher Scientific, Inc.). Cells were cultured on poly-L-lysine coated glass coverslips in 24-well plates and cell differentiation was observed at different time points. Glass coverslips with cells were washed with $0.01 \mathrm{M}$ PBS at 1, 3, 7 and 14 days, respectively, followed by fixation with $4 \%$ PFA for $30 \mathrm{~min}$ at room temperature.

Immunocytochemistry staining. Immunocytochemistry staining was performed following the standard protocol (19). Monoclonal antibodies (EMD Millipore; Billerica, MA, USA) including mouse anti-nestin (1:200; cat. no. MAB353), mouse anti- $\beta$-tubulin III (1:200; cat. no. MAB5564), mouse anti-glial fibrillary acidic protein (GFAP; 1:500; cat. no. MAB360) and mouse anti-oligodendrocytes (1:2,000; cat. no. MAB1580) were used to identify NSCs, neurons and astrocytes, respectively. Polyclonal rabbit anti-srGAP2 (1:50) (17) was raised in Dr Jin's laboratory and used to perform double staining with nestin, $\beta$-tubulin III and GFAP. Primary antibodies were diluted in $0.01 \mathrm{M}$ PBS. Blocking solution contains 

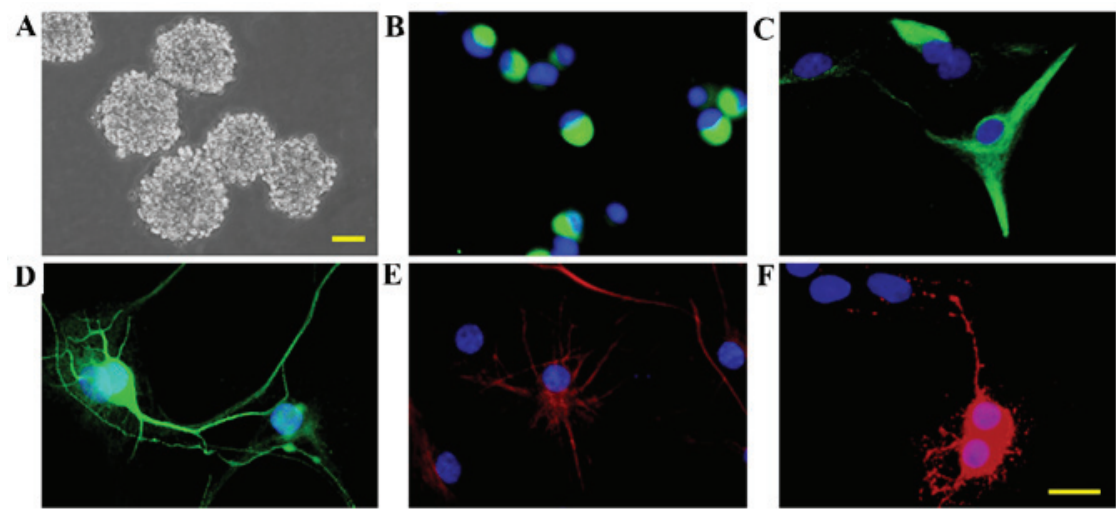

Figure 2. Culture and identification of NSCs. (A) Different sizes of neurospheres developed after 5 days culture in growth medium. (B) Single cells from the neurospheres were nestin ${ }^{+}$. (C) A number of the cells were remained nestin following culture in the differentiation medium for 7 days. NSCs differentiated into (D) $\beta$-tubulin $\mathrm{III}^{+}$neurons, (E) GFAP ${ }^{+}$astrocytes and (F) oligodendrocytes ${ }^{+}$oligodendrocytes. Scale bar $=20 \mu \mathrm{m}$. NSCs, neural stem cells; GFAP, glial fibrillary acidic protein.

A

Nestin

srGAP2

DAPI

Merge
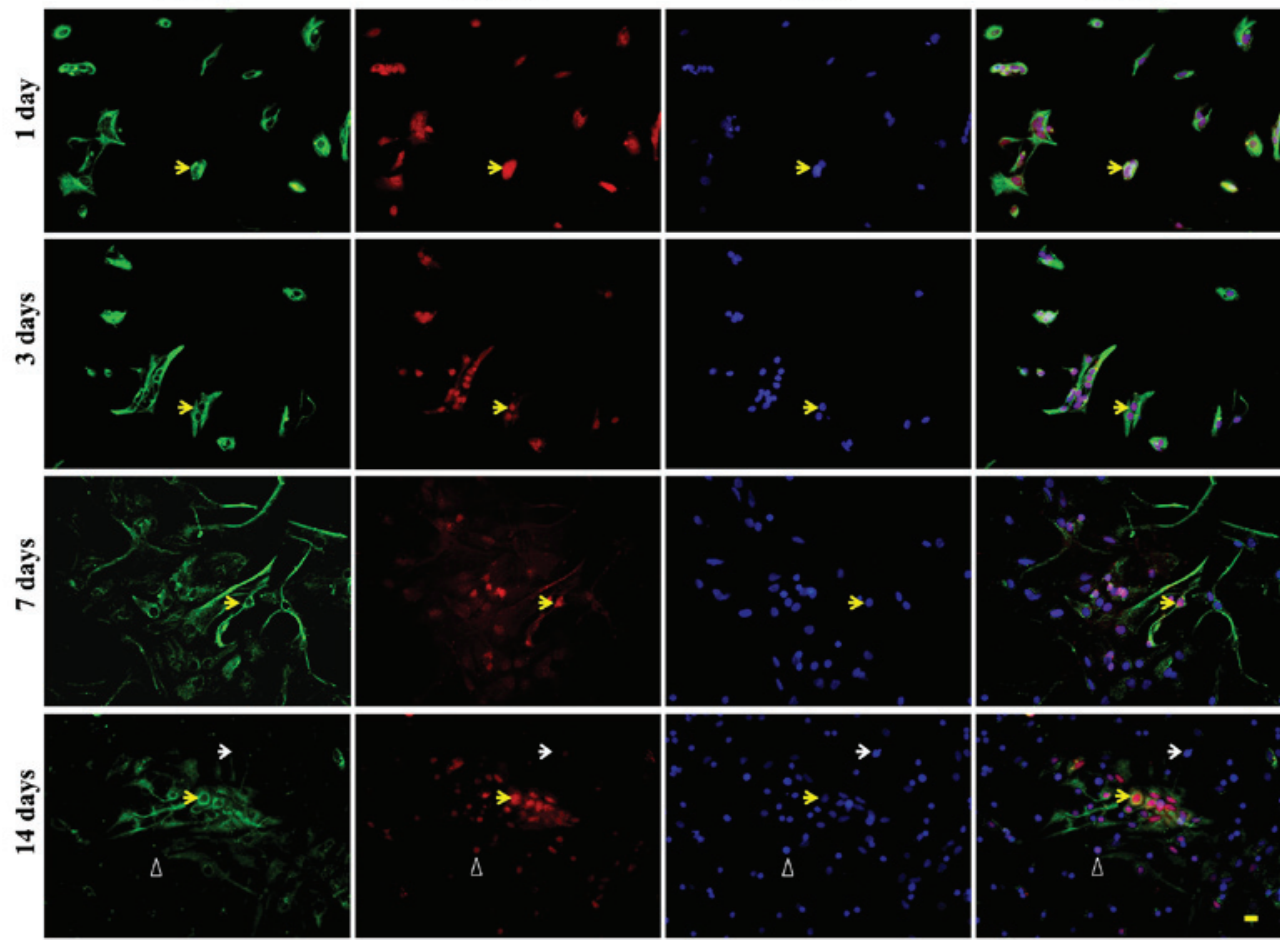

B

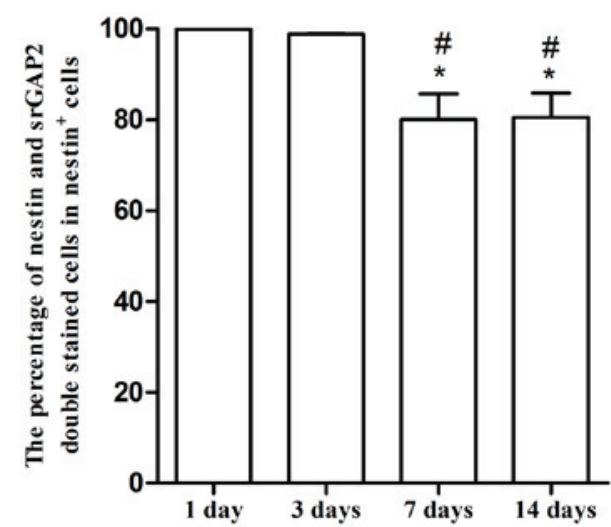

Figure 3. Expression of srGAP2 in nestin ${ }^{+}$cells along NSCs/NPCs differentiation in vitro. (A) In addition to spontaneous differentiation of NSCs/NPCs in vitro, srGAP2 was expressed in the cell nucleus and cytoplasm of nestin ${ }^{+}$cells at 1 and 3 days, however, expression weakens in the cytoplasm at 7 and 14 days. (B) The ratio of srGAP2 $2^{+}$nestin $^{+}$cells to total nestin ${ }^{+}$cells was significantly decreased after 7 days. The values are presented as the mean \pm standard error of the mean. ${ }^{*} \mathrm{P}<0.05$ vs. 1 day, ${ }^{*} \mathrm{P}<0.05$ vs. 3 days. Scale bar $=20 \mu \mathrm{m}$. srGAP2, SLIT-ROBO Rho GTPase-activity protein 2; NSC, neural stem cells; NPC, neural progenitor cells. 

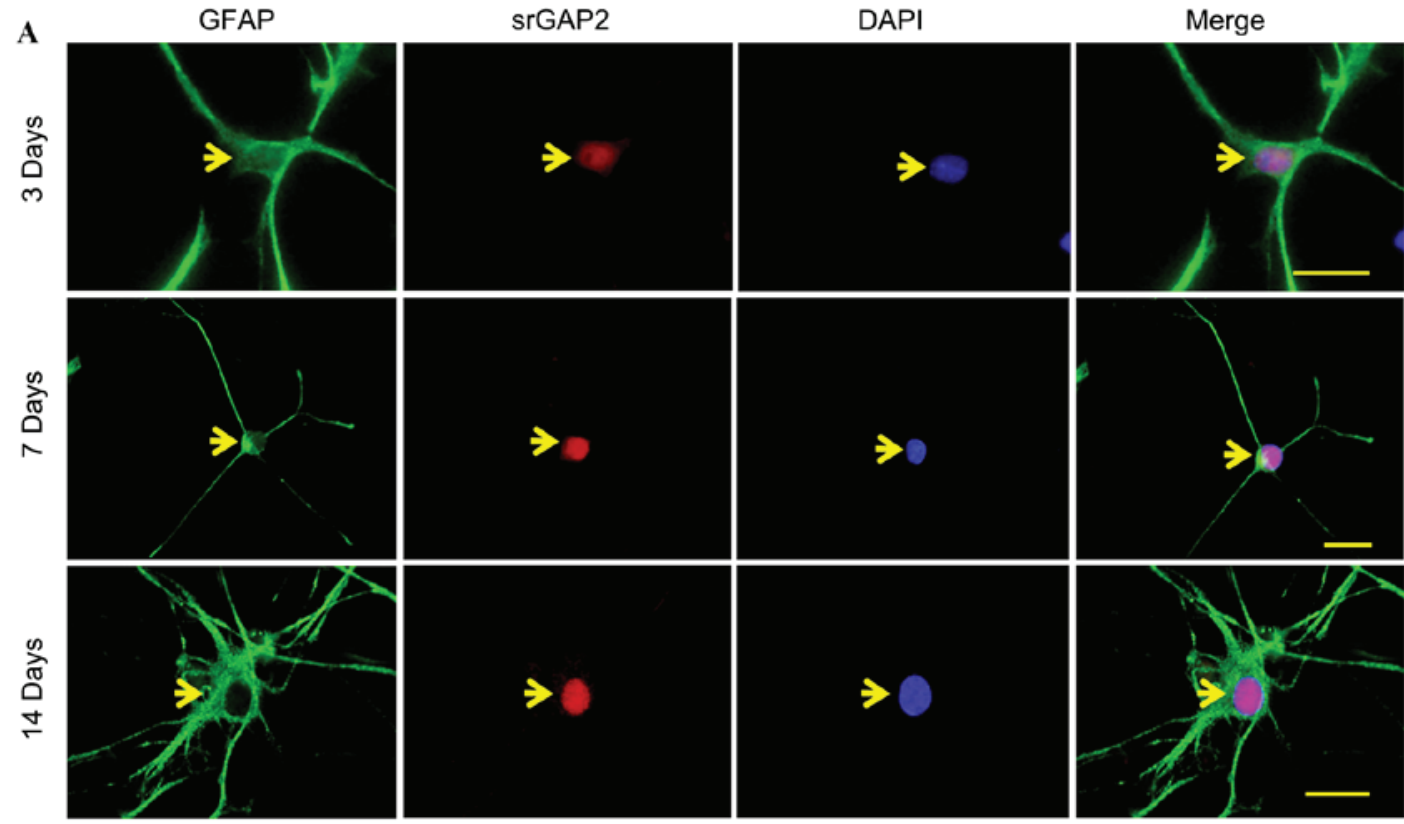

B

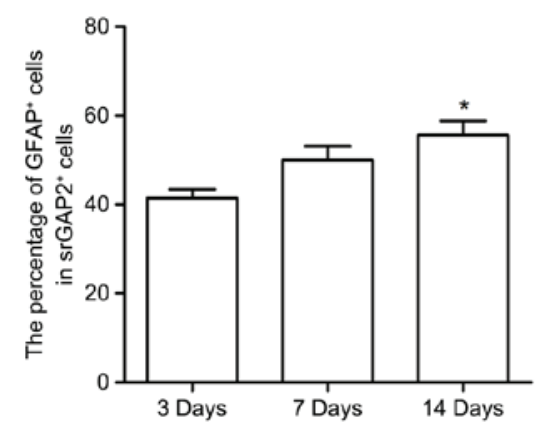

b

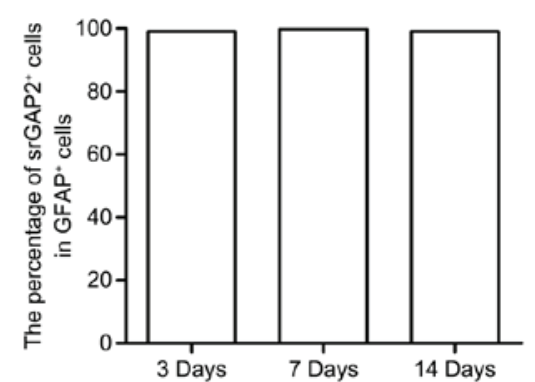

Figure 4. Expression of srGAP2 in $\mathrm{GFAP}^{+}$cells during differentiation in vitro. (A) srGAP2 was predominantly expressed in the nucleus of GFAP ${ }^{+}$cells and slightly increased during the differentiation in vitro (arrows). (B) The ratio of $s r G A P 2^{+} / \mathrm{GFAP}^{+}$cells to total (a) srGAP2 $2^{+}$cells significantly increased, (b) while within the population of $\mathrm{GFAP}^{+}$cells, this ratio was maintained at a similar level. The values are presented as the mean \pm standard error of the mean, ${ }^{*} \mathrm{P}<0.05$ vs. 3 days. Scale bar $=20 \mu \mathrm{m}$. srGAP2, SLIT-ROBO Rho GTPase-activity protein 2; GFAP, glial fibrillary acidic protein.

$10 \%$ normal goat serum (CWBIO, Beijing, China) and $0.3 \%$ Triton X-100 in PBS. Tetramethylrhodamine and fluorescein isothiocyanate-conjugated goat anti-mouse $\mathrm{IgG} /$ anti-rabbit IgG (1:400; CWBIO; cat. nos. CW0152S and CW0113S) were used as secondary antibodies. Cell nuclei were counterstained with DAPI-containing mounting media (Vector Laboratories, Inc., Burlingame, CA, USA) and visualized under a fluorescent microscope (Olympus BX57; Olympus Corporation, Tokyo, Japan) equipped with a DP70 digital camera and the DPManager (DPController) software (Olympus Corporation). For the negative control, the primary antibody was replaced by $0.01 \mathrm{M}$ PBS.

Quantification and statistical analysis. Cell counting was performed using a $20 \mathrm{x}$ objective lens. Immunoreactive cells from 6 random fields ( 2 cover slips, 3 fields from each coverslip) were counted. All the quantitative data were obtained from three independent experiments. They were presented as the mean \pm standard error of the mean, and the statistical analysis was performed with SPSS 13.0 software (SPSS, Inc. Chicago, IL, USA). A one-way analysis of variance followed by Tukey's test was used and $\mathrm{P}<0.05$ was considered to indicate a statistically significant difference.

\section{Results}

Expression of srGAP2 in the subventricular zone (SVZ) in vivo. NSCs/NPCs are distributed in the SVZ and can be stained by GFAP. Double staining immunohistochemistry was used to identify srGAP2 expression in NSCs/NPCs in the SVZ. The results demonstrated that endogenous srGAP2 is detectable in the ventricular zone (VZ)/SVZ of the adult brain and that it co-localizes with GFAP ${ }^{+}$cells in vivo (Fig. 1).

Culture and identification of rat embryonic NSCs. Cells were isolated from the cerebral cortex of rat embryos and cultured in standard growth medium. Neurospheres were observed at 5 DIV (Fig. 2A) and immunocytochemistry staining indicated that the majority of the cells were nestin ${ }^{+}$(Fig. 2B). After 7 days culturing in a differentiation medium, $\beta$-tubulin $\mathrm{III}^{+}$ neurons (Fig. 2D), GFAP ${ }^{+}$astrocytes (Fig. 2E) and oligodendrocytes $^{+}$oligodendrocytes (Fig. 2F) were detected. However a few of the cells did remain nestin ${ }^{+}$(Fig. 2C). The data suggests that the cells cultured were NSCs/NPCs.

Dynamic expression of srGAP2 during in vitro differentiation of NSCs/NPCs. With the spontaneous differentiation of 
A
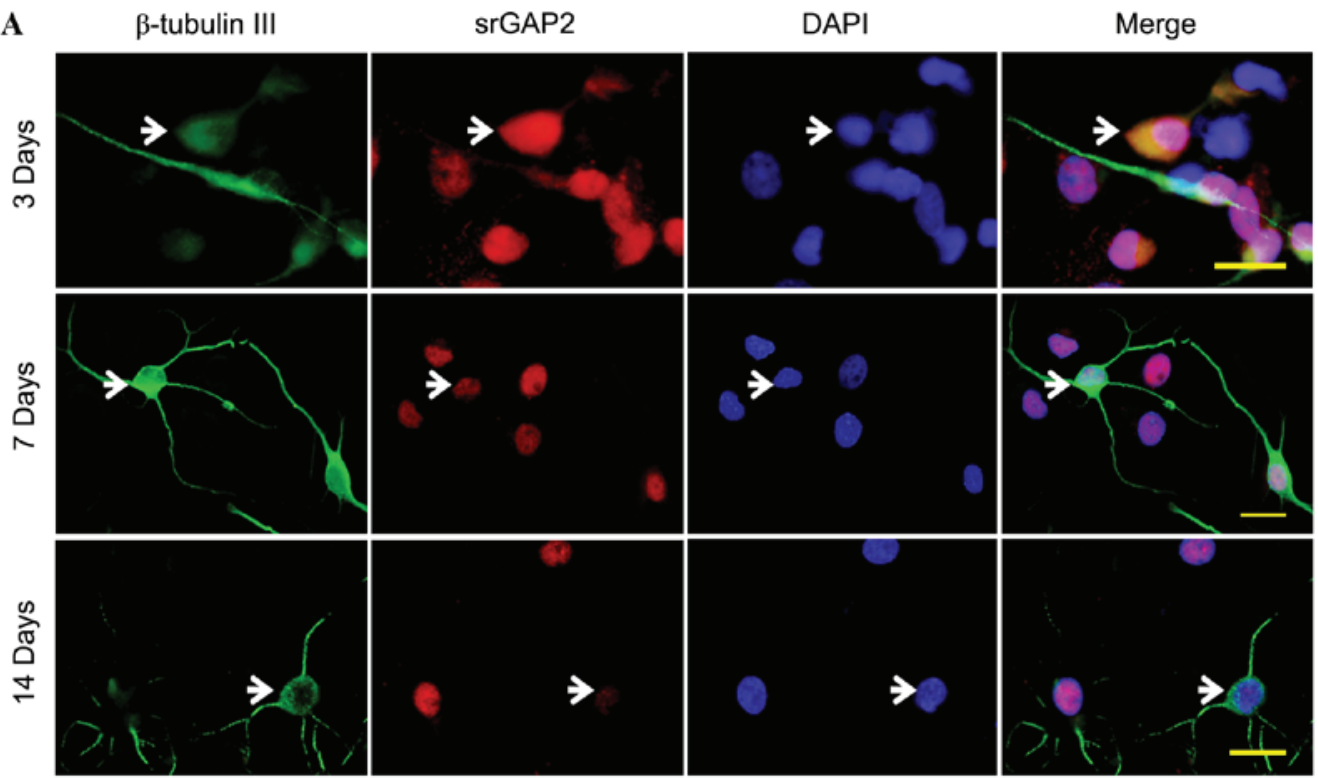

B

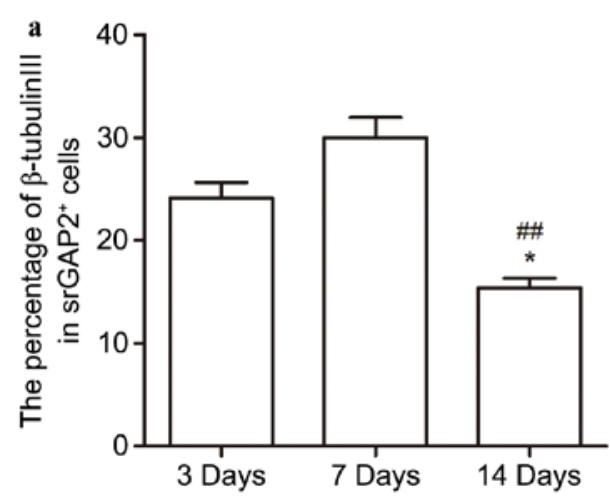

b

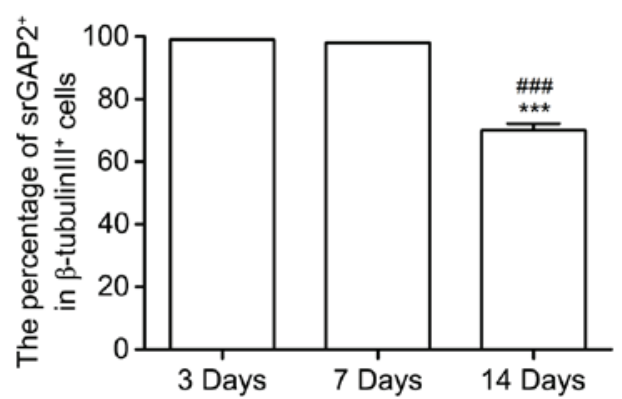

Figure 5. Expression of srGAP2 in $\beta$-tubulin $\mathrm{III}^{+}$cells during differentiation in vitro. (A) srGAP2 was expressed in almost all $\beta$-tubulin $\mathrm{III}^{+}$cells at 3 and 7 days, specifically in the cell nuclei (arrows). (B) However, the percentage of srGAP2 ${ }^{+} / \beta$-tubulin $\mathrm{III}^{+}$from (a) total srGAP2 $2^{+}$cells and (b) total $\beta$-tubulin $\mathrm{III}^{+}$cells was significantly reduced at 14 days and no srGAP2 was observed in the cell cytoplasm at this time. The values are presented as the mean \pm standard error of the mean, ${ }^{*} \mathrm{P}<0.05,{ }^{* * *} \mathrm{P}<0.001$ vs. 3 days, ${ }^{\# \#} \mathrm{P}<0.05,{ }^{\# \# \#} \mathrm{P}<0.001$ vs. 7 days. Scale bar $=20 \mu \mathrm{m}$. srGAP2, SLIT-ROBO Rho GTPase-activity protein 2.

NSCs in vitro, the number of nestin ${ }^{+}$NSCs/NPCs markedly reduced. It was noted that over a period of 7 days, $\sim 56.33 \pm 10.32 \%$ of cells were nestin ${ }^{+}$(data not shown). srGAP2 was predominantly expressed in the cell nucleus (Fig. 3A). The expression of srGAP2 dynamically changed along with the decreased expression of nestin. The ratio of srGAP $^{+} /$nestin $^{+}$cells from the total nestin ${ }^{+}$cells was $\sim 80 \%$ at 7 and 14 days. It was significantly lower than that at 1 and 3 days (almost $100 \%, \mathrm{P}<0.05$ ). No difference was observed between 7 and 14 days (Fig. 3B).

Dynamic expression of srGAP2 in $G F A P^{+}$cells. Along with the differentiation of NSCs/NPCs in vitro, the expression of srGAP2 in $\mathrm{GFAP}^{+}$cells was observed to further elucidate the association between srGAP2 and $\mathrm{GFAP}^{+}$cells. srGAP2 was predominantly expressed in the cell nucleus (Fig. 4A), a similarity also observed in nestin ${ }^{+}$cells. During differentiation, with the slightly enhanced expression of srGAP2 in cell nuclei, the ratio of $\mathrm{srGAP}^{+} / \mathrm{GFAP}^{+}$cells from the total srGAP2 ${ }^{+}$cells significantly increased (Fig. 4Ba; 41.48 \pm 3.37 to $51.02 \pm 5.47 \%$; $\mathrm{P}<0.05)$, while within the population of $\mathrm{GFAP}^{+}$cells, this ratio was maintained at a similar level (Fig. 4Bb).
Altered expression of srGAP2 in $\beta$-tubulin ${ }^{+}$cells in vitro. During culture in the differentiation medium, $\sim 28.9 \pm 3.06 \%$ of NSCs/NPCs differentiated into $\beta$-tubulin $\mathrm{III}^{+}$neuronal progenitors/neurons at 7 days (data not shown). srGAP2 was observed to be expressed in almost all the $\beta$-tubulin $\mathrm{III}^{+}$cells at 3 and 7 days, specifically in the cell nuclei (Fig. 5A and B). However, the percentage of $\mathrm{srGAP} 2^{+} / \beta$-tubulin $\mathrm{III}^{+}$cells compared with the total number of srGAP2 ${ }^{+}$and $\beta$-tubulin $\mathrm{III}^{+}$cells was significantly reduced from $30.02 \pm 3.41$ and almost $100 \%$ on the 3rd day to $15.38 \pm 1.66$ and $68.25 \pm 2.75 \%$ on the 14 th day, $(\mathrm{P}<0.05)$. By contrast, no srGAP2 was observed in the cell cytoplasm of nestin ${ }^{+}$cells on the 14th day.

\section{Discussion}

The current study demonstrated that along with the differentiation of NSCs/NPCs in vitro, expression patterns of srGAP2 changed dynamically. It was expressed predominantly in cell nuclei and markedly decreased with the differentiation of NSCs/NPCs, particularly in $\beta$-tubulin $\mathrm{III}^{+}$neuronal progenitors/neurons.

All members of the srGAP family are widely expressed in the nervous system and work as multifunctional adaptor 
proteins, however, different srGAPs have diverse patterns of expression that are often distinct from each other. This indicates that different srGAPs are likely to be important for different aspects of central nervous system development $(1,4)$. Although srGAP2 is detectable during rat neural development until postnatal day 14 (17), it is notably absent from the site of neurogenesis, but often strongly detected in the region of neuronal migration and differentiation (4). This demonstrates that srGAP2 may not be essential for the production of neuronal precursors. The immunohistochemistry staining of adult rat brain tissue conducted in the present study, demonstrated that srGAP2 is weakly expressed in the SVZ where neurogenesis occurs and that it co-localizes with $\mathrm{GFAP}^{+}$type $\mathrm{B}$ cells, which refers to slowly proliferating cells. Furthermore, it has been confirmed that srGAP2 is involved in neural development, however it may not be involved in neuronal genesis.

Nestin is an intermediate filament protein expressed in dividing cells during the early stages of development. srGAP2 was expressed in nestin ${ }^{+}$NSCs/NPCs throughout their in vitro differentiation. With the downregulation of nestin upon cell differentiation, the ratio of srGAP2/nestin double positive cells compared with total nestin positive cells declined significantly at 7 and 14 days. srGAP2 was expressed predominantly in the cell nucleus. Weak expression of srGAP2 in the cytoplasm markedly reduced after 7 days. This suggested that srGAP2 in cell cytoplasm may be involved in maintaining the stemness, or undifferentiated state, of the NSCs/NPCs. The results of the present study are partially supported by observations in the rat brain described by Yao et al (17).

During neuro- and gliogenesis, nestin is gradually replaced by cell type-specific intermediate filaments, including neurofilaments and GFAP. It was observed that with astrocytic differentiation, srGAP2 was detectable in the cell nucleus only and significantly stronger at 14 days than 3 days. Although the percentage of $\mathrm{srGAP}^{+} / \mathrm{GFAP}^{+}$cells compared with the total $\mathrm{GFAP}^{+}$cells is stable during gliogenesis, it is notable that the ratio of $\mathrm{srGAP}^{+} / \mathrm{GFAP}^{+}$cells to total $\mathrm{srGAP}^{+}$cells is significantly increased at 14 days. This indicated that srGAP2 in the cell nucleus may promote astrocytic differentiation of NSCs/NPCs in vitro.

By contrast, srGAP2 was expressed weakly in the cytoplasm but strongly in the cell nucleus in $\beta$-tubulin $\mathrm{III}^{+}$cells at 3 days. The srGAP2 levels in the cell nucleus were markedly downregulated during neuronal differentiation. This is in contrast with the srGAP2 expression pattern in cultured neurons (17). In addition, the percentage of $\mathrm{srGAP} 2^{+} / \beta$-tubulin $\mathrm{III}^{+}$cells from the total $\beta$-tubulin ${ }^{+}$and the total srGAP2 ${ }^{+}$cells was significantly reduced at 14 days. This suggested that srGAP2 may negatively regulate NSCs/NPCs to differentiate into neurons in vitro. The srGAP3-Rac1 signal pathway may be involved in the attenuation (20).

In conclusion, the present study suggests that srGAP2 is expressed in nestin ${ }^{+}$, $\mathrm{GFAP}^{+}$and $\beta$-tubulin $\mathrm{III}^{+}$cells at different time points throughout the life cycle of the cell, with distinct localization patterns. It suggests that srGAP2 is associated with cellular development of the nervous system. Throughout the differentiation period of the NSCs/NPCs in vitro, srGAP2 was demonstrated to be expressed at varying levels in different cell types. The translocation of srGAP2 in the cytoplasm and cell nucleus in different cell types may function as a director during cell fate decision. srGAP2 levels in the cell cytoplasm are involved in maintaining the stemness, or, undifferentiated state of NSCs/NPCs, whilst in the cell nucleus, it may promote astrocytic differentiation and attenuate neuronal differentiation of the NSCs/NPCs in vitro. As Rho-GTPases are known to have diverse roles in neurogenesis, srGAP2 will have different functional consequences. In addition, the srGAP2 gene has recently been implicated in a severe neurodevelopmental syndrome that causes early infantile epileptic encepholopathy and srGAP2 knockout mice are prone to epileptic seizures. Thus, srGAP2 may also be associated with neuronal functions. Further investigation into the underlying mechanisms of the diverse functions of srGAP2 in different cell types and different developmental periods is required.

\section{Acknowledgements}

The present study was supported by grants from the National Natural Science Foundation of China (grant nos. 31070943, 81070998, 31271151 and 30960107) and partially supported by the State Key Laboratory of Neuroscience, Shanghai Institutes for Biological Sciences, Chinese Academy of Sciences (grant no. SKLN-210204).

\section{References}

1. Wong K, Ren XR, Huang YZ, Xie Y, Liu G, Saito H, Tang H, Wen L, Brady-Kalnay SM, Mei L, et al: Signal transduction in neuronal migration: Roles of GTPase activating proteins and the small GTPase Cdc42 in the Slit-Robo pathway. Cell 107: 209-221, 2001.

2. Aspenström P: Roles of F-BAR/PCH proteins in the regulation of membrane dynamics and actin reorganization. Int Rev Cell Mol Biol 272: 1-31, 2009.

3. Ypsilanti AR, Zagar Y and Chédotal A: Moving away from the midline: New developments for Slit and Robo. Development 137: 1939-1952, 2010

4. Bacon C, Endris V and Rappold G: Dynamic expression of the Slit-Robo GTPase activating protein genes during development of the murine nervous system. J Comp Neurol 513: 224-236, 2009.

5. Guerrier S, Coutinho-Budd J, Sassa T, Gresset A, Jordan NV, Chen K, Jin WL, Frost A and Polleux F: The F-BAR domain of srGAP2 induces membrane protrusions required for neuronal migration and morphogenesis. Cell 138: 990-1004, 2009.

6. Charrier C, Joshi K, Coutinho-Budd J, Kim JE, Lambert N, de Marchena J, Jin WL, Vanderhaeghen P, Ghosh A, Sassa T and Polleux F: Inhibition of SRGAP2 function by its human-specific paralogs induces neoteny during spine maturation. Cell 149: 923-935, 2012.

7. Soderling SH, Guire ES, Kaech S, White J, Zhang F, Schutz K, Langeberg LK, Banker G, Raber J and Scott JD: A WAVE-1 and WRP signaling complex regulates spine density, synaptic plasticity, and memory. J Neurosci 27: 355-365, 2007.

8. Lu H, Jiao Q, Wang Y, Yang Z, Feng M, Wang L, Chen X, Jin W and Liu Y: The mental retardation-associated protein srGAP3 regulates survival, proliferation, and differentiation of rat embryonic neural stem/progenitor cells. Stem Cells Dev 22: 1709-1716, 2013.

9. Soderling SH, Binns KL, Wayman GA, Davee SM, Ong SH, Pawson T and Scott JD: The WRP component of the WAVE-1 complex attenuates Rac-mediated signalling. Nat Cell Biol 4: 970-975, 2002.

10. Saitsu H, Osaka H, Sugiyama S, Kurosawa K, Mizuguchi T, Nishiyama K, Nishimura A, Tsurusaki Y, Doi H, Miyake N, et al: Early infantile epileptic encephalopathy associated with the disrupted gene encoding Slit-Robo Rho GTPase activating protein 2 (SRGAP2). Am J Med Genet A 158A: 199-205, 2012. 
11. Carlson BR, Lloyd KE, Kruszewski A, Kim IH, Rodriguiz RM, Heindel C, Faytell M, Dudek SM, Wetsel WC and Soderling SH: WRP/srGAP3 facilitates the initiation of spine development by an inverse F-BAR domain, and its loss impairs long-term memory. J Neurosci 31: 2447-2460, 2011.

12. Kim IH, Carlson BR, Heindel CC, Kim H and Soderling SH: Disruption of wave-associated Rac GTPase-activating protein (Wrp) leads to abnormal adult neural progenitor migration associated with hydrocephalus. J Biol Chem 287: 39263-39274, 2012.

13. Waltereit R, Leimer U, von Bohlen Und Halbach O, Panke J, Hölter SM, Garrett L, Wittig K, Schneider M, Schmitt C, Calzada-Wack J, et al: Srgap3\% mice present a neurodevelopmental disorder with schizophrenia-related intermediate phenotypes. FASEB J 26: 4418-4428, 2012.

14. Parati EA, Pozzi S, Ottolina A, Onofrj M, Bez A and Pagano SF: Neural stem cells: An overview. J Endocrinol Invest 27 (6 Suppl): S64-S67, 2004.

15. Price J and Williams BP: Neural stem cells. Curr Opin Neurobiol 11: 564-567, 2001
16. Jiao Q, Xie WL, Wang YY, Chen XL, Yang PB, Zhang PB, Tan J, Lu HX and Liu Y: Spatial relationship between NSCs/ NPCs and microvessels in rat brain along prenatal and postnatal development. Int J Dev Neurosci 31: 280-285, 2013.

17. Yao Q, Jin WL, Wang Y and Ju G: Regulated shuttling of Slit-Robo-GTPase activating proteins between nucleus and cytoplasm during brain development. Cell Mol Neurobiol 28: 205-221, 2008.

18. Gage FH, Coates PW, Palmer TD, Kuhn HG, Fisher LJ, Suhonen JO, Peterson DA, Suhr ST and Ray J: Survival and differentiation of adult neuronal progenitor cells transplanted to the adult brain. Proc Natl Acad Sci USA 92: 11879-11883, 1995.

19. Lu HX, Hao ZM, Jiao Q, Xie WL, Zhang JF, Lu YF, Cai M, Wang YY, Yang ZQ, Parker T and Liu Y: Neurotrophin-3 gene transduction of mouse neural stem cells promotes proliferation and neuronal differentiation in organotypic hippocampal slice cultures. Med Sci Monit 17: BR305-BR311, 2011.

20. Ma Y, Mi YJ, Dai YK, Fu HL, Cui DX and Jin WL: The inverse F-BAR domain protein srGAP2 acts through srGAP3 to modulate neuronal differentiation and neurite outgrowth of mouse neuroblastoma cells. PLoS One 8: e57865, 2013. 